\title{
OBSERVATIONS OF "EFFECTIVE" TRANSVERSE BEAM-SIZE INSTABILITIES FOR A HIGH CURRENT PER BUNCH FILL PATTEREN IN THE APS STORAGE RING*
}

\author{
A. H. Lumpkin, L. Emery and B. X. Yang
}

Advanced Photon Source, Argonne National Laboratory, Argonne, IL 6043

\begin{abstract}
The X-ray pinhole camera diagnostics on the Advanced Photon Source (APS) storage ring have recorded an "effective" transverse beam size instability during operations with a sextuplet plus 22 singlets fill pattern. These instabilities were not observed with the sextuplet plus 25 triplets fill pattern that has been the standard fill pattern in FY' 98 . The instability threshold is at $82-85 \mathrm{~mA}$ with positrons. The features include an increased average (few seconds) transverse size both horizontally and vertically for stored currents above the threshold with a correlated effect on the beam lifetime. The horizontal transverse emittance is $25-30 \%$ larger at $100 \mathrm{~mA}$ than below the threshold. There is a related horizontal beam centroid motion as well, but this does not explain the vertical size change nor the lifetime effect. Complementary data were also taken with the diagnostic undulator, and a similar threshold effect on divergence was observed. The cross-comparison of the data and possible mechanisms will be presented.
\end{abstract}

\section{INTRODUCTION}

Operations of the Advanced Photon Source (APS) storage ring have included two fill patterns in the last year: one with a bunch sextuplet followed by 25 bunch triplets that were spaced by $140 \mathrm{~ns}$ and the other with the sextuplet followed by 22 singlets spaced 100 ns apart. In both cases the total stored current in the ring at the end of the fill is the nominal $100 \mathrm{~mA}$, but in the singlets case the charge per bunch is about three times higher than in each bunch of the triplets. In the singlets-fill case our $\mathrm{x}$-ray pinhole camera diagnostics were used to identify two instabilities that result in increases in averaged transverse beam sizes. One instability occurs only near the maximum beam current in the present conditions, and the horizontal beam size at the dispersive point is dramatically increased. The other is also present down to the threshold of $82-85 \mathrm{~mA}$. Several cross-comparisons of results from both the dispersive and nondispersive bending magnet source points, the diagnostics undulator, and the streak camera bunch length data were used to separate the features of the two instabilities. These features are consistent with a transverse instability with the $82-85 \mathrm{~mA}$ threshold and a longitudinal instability at the top of the fill. The first is

*Work supported by the U.S. Department of Energy, Office of Basic Energy Sciences, under Contract No. W-31-109-ENG-38. sensitive to changes in the sets of sextupol currents (chromaticity) and the second to changes in the rf cavity temperature setpoints (HOMs). Representative examples of the different types of data will be presented. The observations were initially performed with stored positrons, but the basic features persisted with the change to electrons in October 1998.

\section{EXPERIMENTAL BACKGROUND}

The APS storage ring utilizes a $7-\mathrm{GeV}$ positron or electron beam (since Oct. 1998) circulating in a 1104-m circumference ring. Normal stored beam currents are 100 $\mathrm{mA}$ with a natural emittance, $\varepsilon=7.9 \pm 1.1 \mathrm{~nm}$ rad. The baseline vertical coupling was $10 \%$, but we now generally run at the $1-2 \%$ level. The standard fill pattern involves a sextuplet (each of similar intensity) totaling $10 \mathrm{~mA}$ of stored beam current. The other $90 \mathrm{~mA}$ are distributed in 25 triplets spaced $140 \mathrm{~ns}$ apart in the ring. In a special user mode we have run $-15 \mathrm{~mA}$ in the sextuplet and 85 $\mathrm{mA}$ distributed in 22 singlets that are $100 \mathrm{~ns}$ apart. It is this latter fill pattern, with about three times the charge per bunch that has exhibited the "effective" transverse beam size growth. These phenomena have been detected with photon diagnostics, If BPMs, and the tune measurement system. This paper will concentrate on the photon diagnostics results.

The photon diagnostics are located in one of the 40 sectors of the APS [1-3]. We use radiation from bending magnets and a diagnostics undulator as shown in Fig. 1. For the dipole magnet source at a dispersive point in the lattice, both $\mathrm{x}$-ray synchrotron radiation (XSR) and optical synchrotron radiation (OSR) techniques are used. An in-tunnel $x$-ray pinhole camera includes a remotely controlled four-jaw aperture at $9.1 \mathrm{~m}$ from the source, a $\mathrm{CdWO}_{4}$ or $\mathrm{YAG}: \mathrm{Ce}$ converter crystal at $17 \mathrm{~m}$ from the source, and a charge-coupled device (CCD) camera. Effective spatial resolutions of about $25 \mu \mathrm{m}(\sigma)$ are estimated. The video is digitized by a Data Cube MaxVideo-200 (MV200) unit, and the digital results are identified as process variables (PVs) for the EPICS platform. Data logging of beam size, centroid, and emittance can thus be done on a 24-hour period. The OSR is transported out of the tunnel to an optics station where a CCD camera, a Stanford Computer Optics (SCO) Quik05 gated camera, and a Hamamatsu C5680 dual-sweep streak camera are available. The synchroscan unit is phase locked to $117.3 \mathrm{MHz}$, the third subharmonic of the SR if 


\section{DISCLAIMER}

This report was prepared as an account of work sponsored by an agency of the United States Government. Neither the United States Government nor any agency thereof, nor any of their employees, make any warranty, express or implied, or assumes any legal liability or responsibility for the accuracy, completeness, or usefulness of any information, apparatus, product, or process disclosed, or represents that its use would not infringe privately owned rights. Reference herein to any specific commercial product, process, or service by trade name, trademark, manufacturer, or otherwise does not necessarily constitute or imply its endorsement, recommendation, or favoring by the United States Government or any agency thereof. The views and opinions of authors expressed herein do not necessarily state or reflect those of the United States Government or any agency thereof. 


\section{DISCLAIMER}

Portions of this document may be illegible in electronic image products. Images are produced from the best available original document. 


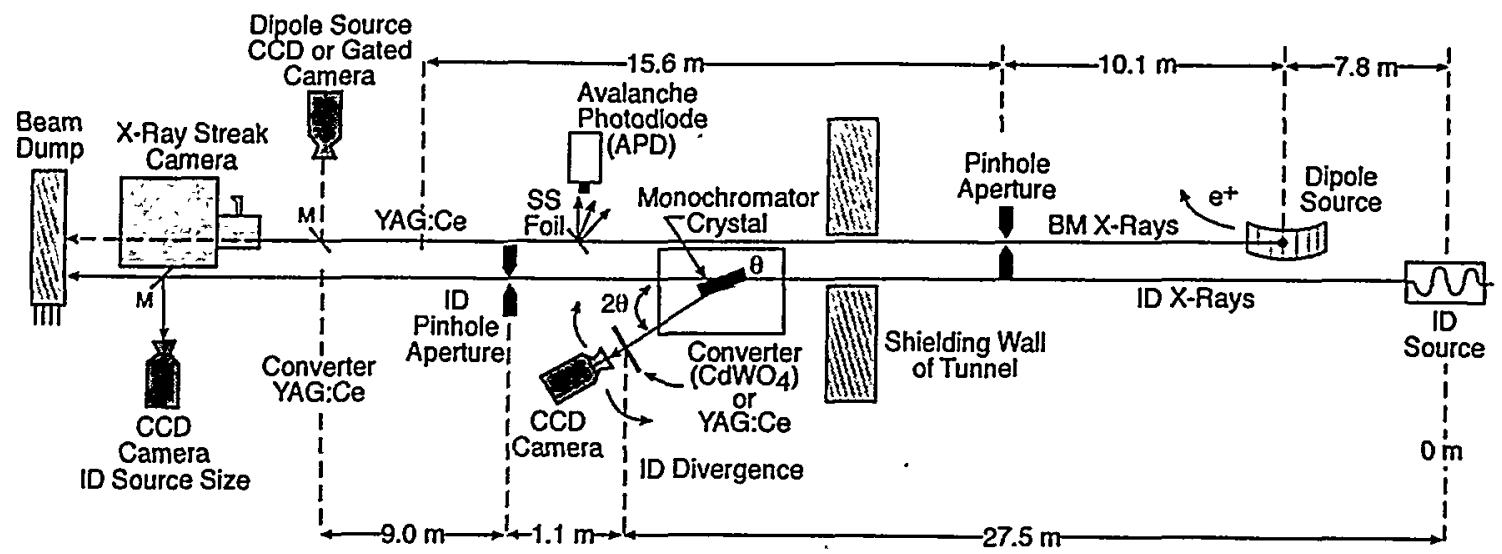

Figure 1: A schematic view of the dipole and diagnostics undulator beamline. On the undulator line the monochromator and pinhole are indicated. On the dipole source the aperture is in the tunnel, and the converter and camera are indicated.

master oscillator. In these studies the streak camera was used to monitor changes in average stored beam bunch length as a function of current. The streak images also were processed by a second MV200 and tracked.

Additionally, the nearby diagnostics undulator and lowdispersion-point dipole source beamline were used as warranted. As schematically shown in Fig. 1, the undulator radiation (UR) is used with the single-crystal monochromator for divergence information and the subsequent four-jaw aperture for horizontal beam-size information [4,5]. The divergence resolution is about 2.6 $\mu \mathrm{rad}(\sigma)$ and the pinhole resolution is about $60 \mu \mathrm{m}(\sigma)$.

\section{EXPERIMENTAL RESULTS AND DISCUSSION}

In this section representative examples of results from the different sources and for the two principal instabilities will be presented. The EPICS data logger and a second MV200 allowed us to select different image sources while keeping the $\mathrm{x}$-ray pinhole camera as a reference for each 12-hour stored beam decay period.

\subsection{Transverse Instability}

The basic observation in the $x$-ray pinhole data is the increased averaged horizontal beam size for some current levels. We attribute this size increase to both a measured centroid oscillation at the fractional beta from tune of 0.20 and an intrinsic size increase of some kind. In Fig. 2 the effects are plotted versus stored positron beam current. Both the horizontal beam size and emittance in Fig. $2 \mathrm{a}$ and $2 \mathrm{~b}$, respectively, are larger above $\sim 85 \mathrm{~mA}$. There is a correlated change in the slope of beam lifetime versus current also at $\sim 85 \mathrm{~mA}$ as seen in Fig. $2 \mathrm{c}$. In fact, the correlated change in lifetime seen in Fig. 2 is why we do not attribute only a centroid motion to the observed increase in transverse horizontal size. The only effect in the streak camera data was the expected gradual decrease in bunch length with decrease in single-bunch current.
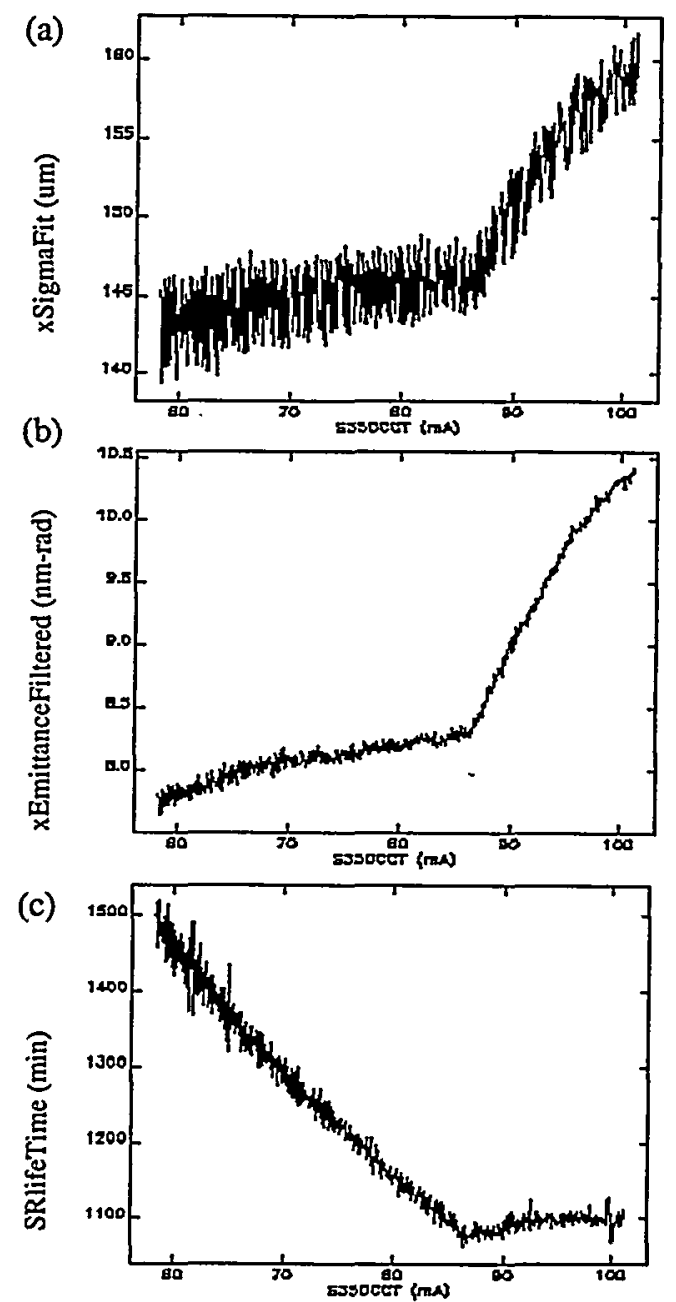

Figure 2: Plots of beam a) horizontal size (dispersive point) and b) horizontal emittance, and c) stored beam lifetime versus stored beam current. There is a correlated change in the respective slopes at $\sim 85 \mathrm{~mA}$ of stored positrons.

During the stored electron beam runs in Dec. 1998 the $x$-ray pinhole camera data were compared to the ID divergence data, the ID pinhole data, lifetime data, and 
the streak camera. Correlated changes were seen in the first two horizontal transverse sizes, as shown in Fig. 3, and also with the lifetime.
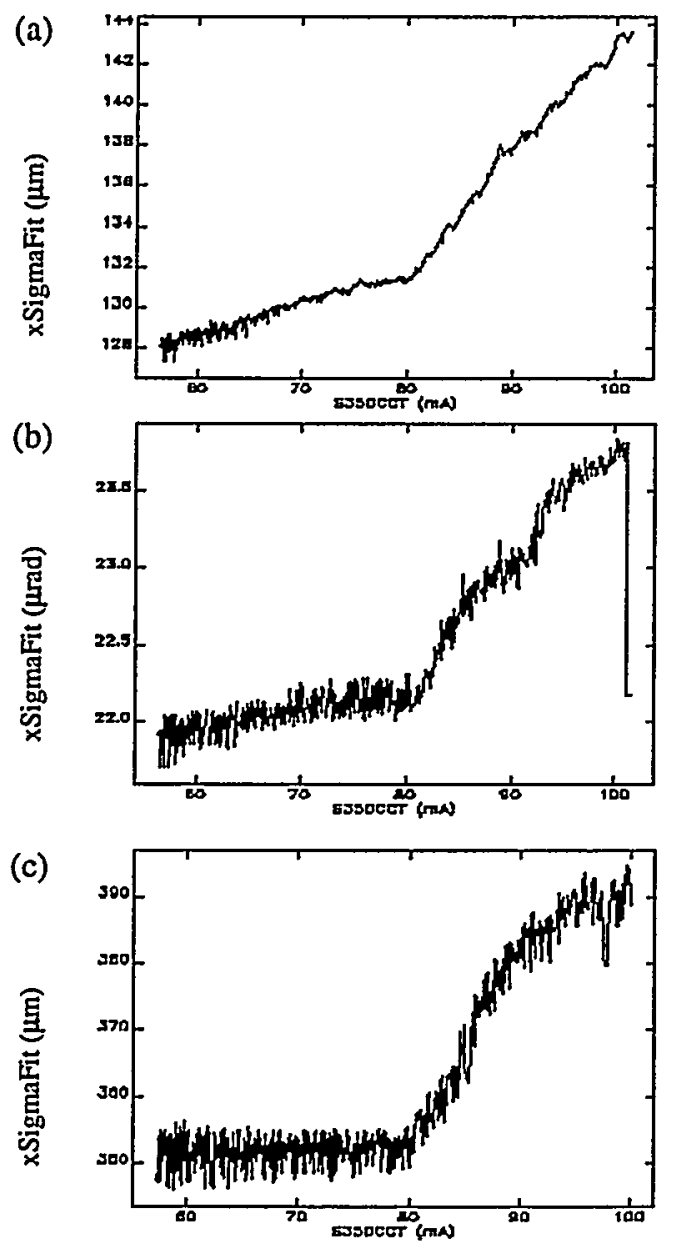

Figure 3: Plots of the variation with current of stored electron beam a) horizontal size at a dispersive point, b) horizontal divergence, and c) ID source point size. All have the increased values above $85 \mathrm{~mA}$.

\subsection{Longitudinal Instability}

In this case at currents around $101 \mathrm{~mA}$, the observed horizontal beam size shows growth from 140 to $260 \mu \mathrm{m}$ as shown in Fig. 4a. In Fig. $4 \mathrm{~b}$ the beam bunch length change from $35 \mathrm{ps}$ to $70 \mathrm{ps}$ is shown over the same 10min period. An effect in longitudinal phase space is clearly supported.

Additionally, the nature of the bunch length blurring is related to a phase instability detected by a dual-sweep streak camera. A synchrotron oscillation can also develop over the many turns at $1.8 \mathrm{kHz}$. The superposition of the two effects gives the $30-\mathrm{ms}$ averaged bunch-length change from $35 \mathrm{ps}$ to $70 \mathrm{ps}(\sigma)$.

The magnitude of this effect was sensitive to the if cavity temperature and was reduced by changing the rf cavity temperature setpoint. It is suspected that the higher-order modes (HOMs) are strongly temperature dependent and are contributors to the effects observed.
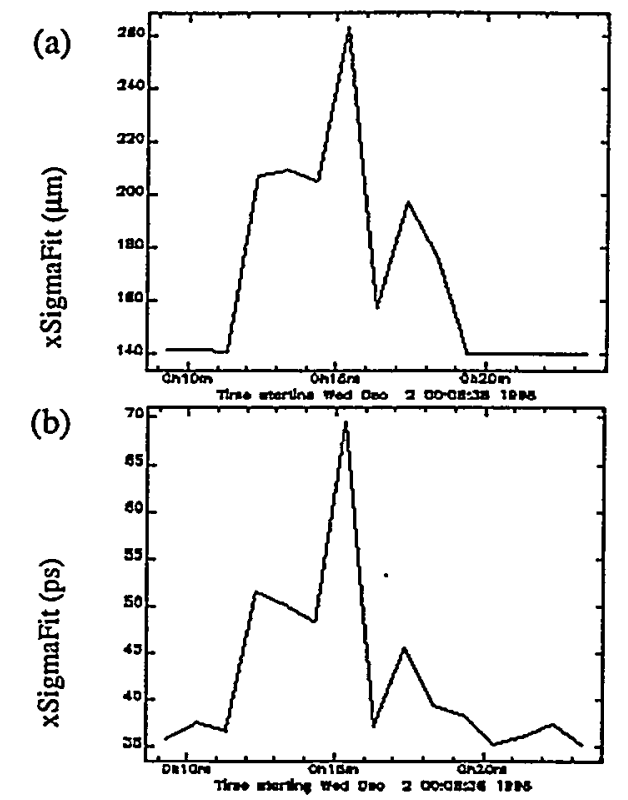

Figure 4: Plots of the correlated change in a) horizontal beam size at a dispersive point and $b$ ) the beam bunch length near 101-mA stored beam currents. These are consistent with a longitudinal instability.

\section{SUMMMARY}

In summary, we have used the correlated observations of beam transverse size, beam divergence, and bunch length to sort the features of the two instabilities that cause an increase in effective beam size. The evidence is consistent with a transverse instability with a threshold at $80-85 \mathrm{~mA}$ that can be controlled with increased chromaticity. The longitudinal instability near $101 \mathrm{~mA}$ (at present) is controlled by if cavity temperature setpoints. Further experiments with a gated camera to search for horizontal quadrupolar effects or energy centroid shifts may be of interest. We are now in position to provide a more stable singlets-fill beam for the users, and this was successfully done in March 1999.

\section{REFERENCES}

[1] A.H. Lumpkin and B.X. Yang, "Status of the Synchrotron Monitors for the APS Facility Rings," Proc. of the 1995 PAC, IEEE, Vol. 4, p. 2470 (1996).

[2] B.X. Yang and A.H. Lumpkin, "Initial Time-Resolved Particle Beam Profile Measurements at the Advanced Photon Source," SRI95, Rev. Sci. Instrum. 67, 1996.

[3] A.H. Lumpkin et al., "Recent Observations on the APS Storage Ring Using Synchrotron Radiation Monitors," Proc. of EPAC'98, Stockholm, p. 1556 (1999).

[4] B.X. Yang et al., "Status of the APS Diagnostics Undulator Beamline," Proc. of the 1997 PAC, IEEE, Vancouver, B.C., (1999).

[5] B.X. Yang and A.H. Lumpkin, "Simultaneous Measurement of Electron Beam Size and Divergence with an Undulator," these proceedings. 